\title{
Optical coherence tomography for the early detection of colorectal dysplasia and cancer: validation in a murine model
}

\author{
Jian Ding ${ }^{1 \#}$, Qiu Li ${ }^{1 \#}$, Jiewen Lin ${ }^{2}$, Shanshan $\mathrm{He}^{1}$, Weiqiang Chen ${ }^{2}$, Qiyong $\mathrm{He}^{1}$, Qiukun Zhang ${ }^{2}$, \\ Jintong Chen ${ }^{1}$, Ting $\mathrm{Wu}^{1}$, Shuncong Zhong ${ }^{2}$, Dan $\mathrm{Li}^{3}$ \\ ${ }^{1}$ Digestive Department, the First Affiliated Hospital of Fujian Medical University, Fuzhou, China; ${ }^{2}$ Laboratory of Optics, Terahertz and Non- \\ Destructive Testing, School of Mechanical Engineering and Automation, Fuzhou University, Fuzhou, China; ${ }^{3}$ Department of Gastroenterology, \\ Union Hospital, Fujian Medical University, Fuzhou, China \\ "These authors contributed equally to this work.
}

Correspondence to: Dan Li. Department of Gastroenterology, Union Hospital, Fujian Medical University, 29 Xinquan Road, Fuzhou 350001 , China. Email: doctorlidan@163.com; Shuncong Zhong. Laboratory of Optics, Terahertz and Non-Destructive Testing, School of Mechanical Engineering and Automation, Fuzhou University, Fuzhou 350108, China. Email: zhongshuncong@hotmail.com.

Background: There is an urgent need to develop a non-invasive imaging technique for detecting colorectal dysplasia and cancer. Technology for early and real-time microscopic assessments to select the most representative biopsy sites would also be of clinical value. In this study, we explored the sensitivity of optical coherence tomography (OCT) in detecting local lesions to demonstrate its potential for the early detection of colorectal dysplasia and cancer.

Methods: An azoxymethane/dextran sodium sulfate mouse model of colorectal carcinogenesis was utilized. Mice were imaged by OCT, and colorectal tissue sections were observed with hematoxylin and eosin staining. The results of the parallel analyses were compared to evaluate the performance of OCT in imaging and early screening of colorectal lesions.

Results: Dysplasia and cancer could be distinguished from normal colon tissues based on the OCT images. However, simple morphological changes observed in the OCT images were not sufficient to distinguish different degrees of dysplasia or distinguish dysplasia from cancerous tissues. The Youden index and diagnostic efficiency of OCT for colorectal dysplasia and cancer were $62.50 \%$ and $82.14 \%$, respectively, while the sensitivity and specificity were $87.50 \%$ and $75.00 \%$, respectively. Further, the positive and negative predictive values were $82.35 \%$ and $81.82 \%$, respectively.

Conclusions: Based on our findings, we predict that OCT is a promising non-invasive imaging technique that can offer excellent positive detection rates and diagnostic accuracy for early colorectal dysplasia and cancer. This technique is expected to be valuable in realizing real-time qualitative analysis and guided targeted biopsy.

Keywords: Hematoxylin-eosin staining (HE staining); colorectal carcinoma; dysplasia; tomography, optical coherence

Submitted Jan 05, 2020. Accepted for publication Sep 15, 2020.

doi: 10.21037 /qims-20-13

View this article at: http://dx.doi.org/10.21037/qims-20-13 


\section{Introduction}

Optical coherence tomography (OCT) is an optical imaging modality used to obtained high-resolution, cross-sectional, subsurface tomographic images of the microstructures in biological tissues. The imaging principle of OCT is somewhat analogous to that of ultrasound, except that it relies on near-infrared light waves rather than acoustic waves (1). The resolution of OCT in vivo is 10-25 times better than that of high-frequency ultrasound imaging. Furthermore, the imaging depth of OCT is $1-3 \mathrm{~mm}$, which is comparable to the sampling range of conventional biopsies (2). Previous studies have demonstrated that diagnostic results obtained by OCT are almost consistent with those of pathology examinations, validating this imaging method as an "optical biopsy" technique (2).

Colonoscopy with biopsy remains the gold standard for assessing colorectal lesions. However, it cannot provide sufficient resolution to identify atypical, non-protruding lesions, and early-stage lesions that lack microvascular structures or scattered blood vessels. Fortunately, OCT offers an advantage by enabling subsurface tomographic imaging, making it possible to differentiate the atypical and early-stage neoplastic lesions from normal tissue more accurately. Compared with virtual chromoendoscopy and confocal endomicroscopy $(2,3)$, OCT is one of the most prospective and efficient techniques for identifying early dysplasia and carcinoma. Hence, in this study, we investigate the sensitivity of OCT for detecting colorectal dysplasia and cancer.

\section{Methods}

\section{AOM/DSS model of colorectal carcinogenesis}

Healthy female BALB/c mice [8 weeks of age, 22-25 g, specific pathogen free (SPF) grade] were purchased from Wu's Laboratory Animal Center (Minhou County, Fuzhou, Fujian, China). All animals were maintained under a 12-hour light/dark cycle at $22 \pm 2{ }^{\circ} \mathrm{C}$ with $(55 \pm 5) \%$ humidity and fed a standard diet ad libitum. The weight of each animal was recorded weekly. Experiments were performed under a project license (No.: FJMU IACUC 2019-0069) granted by the Institutional Animal Care and Use Committee (IACUC) of Fujian Medical University, in compliance with IACUC institutional guidelines for the care and use of animals.

Following Neufert's method (4), the mice were randomly divided into a control group (12 mice) and a colorectal cancer group (20 mice); the animals in both groups had similar physiological parameters. The mice in the control group received one intraperitoneal injection of normal saline $(10 \mathrm{mg} / \mathrm{kg}$ body weight) and were subsequently allowed to drink distilled water freely. The mice in the colorectal cancer group were subjected to one intraperitoneal injection of azoxymethane (AOM) $(10 \mathrm{mg} / \mathrm{kg}$ body weight) and three exposure cycles to dextran sodium sulfate (DSS), each cycle involving the addition of $2.5 \%$ DSS to the drinking water for 7 days, after which the DSS-doped water was replaced with distilled water for 14 days.

\section{$O C T$}

A frequency-domain OCT imaging system with a center wavelength of $1,000 \mathrm{~nm}$, an axial resolution of $4 \mu \mathrm{m}$, and a scanning frequency of $16 \mathrm{kHz}$ was used in this experiment (Figure 1) (5). The beam was divided evenly into the sample and the reference arms of a fiber-based Michelson interferometer $(6,7)$. The sample beam was routed to the sample via mirrors driven by two galvanometers to allow the beam to be scanned in two directions. When the scanning beam was focused on the sample, a MachZehnder interferometer collected the laser light reflected by the sample and generated a frequency-clock signal with a uniformly spaced optical frequency to sample the OCT signal. Depth-resolved tomography was achieved by applying a fast Fourier transform to the interference fringes. Then, the data were processed and used to reconstruct three-dimensional (3D) images.

\section{OCT imaging procedure}

Mice were fasted from solid food for 24 hours then sacrificed by inhalation of anesthesia with isoflurane. The colon of each mouse was harvested, and a $0.5 \mathrm{~cm}$-long colorectal tissue section $2 \mathrm{~cm}$ away from the anal margin was removed. The tissue samples were flushed with isotonic saline, longitudinally incised, dried on filter paper, and laid on glass slides. The samples were placed in the scanning window of an OCT instrument and scanned within 15 minutes of preparation.

Currently, there are no unified and approved diagnostic criteria to diagnose colorectal dysplasia and cancer using OCT. However, several studies have demonstrated that colorectal dysplasia and cancer are characterized by loss of tissue organization and reduced light scattering (8-10). In OCT scanning, dysplasia and cancer are recognized as "the same lesion". In this study, we aimed to demonstrate that lesions can be distinguished from normal tissue. OCT 


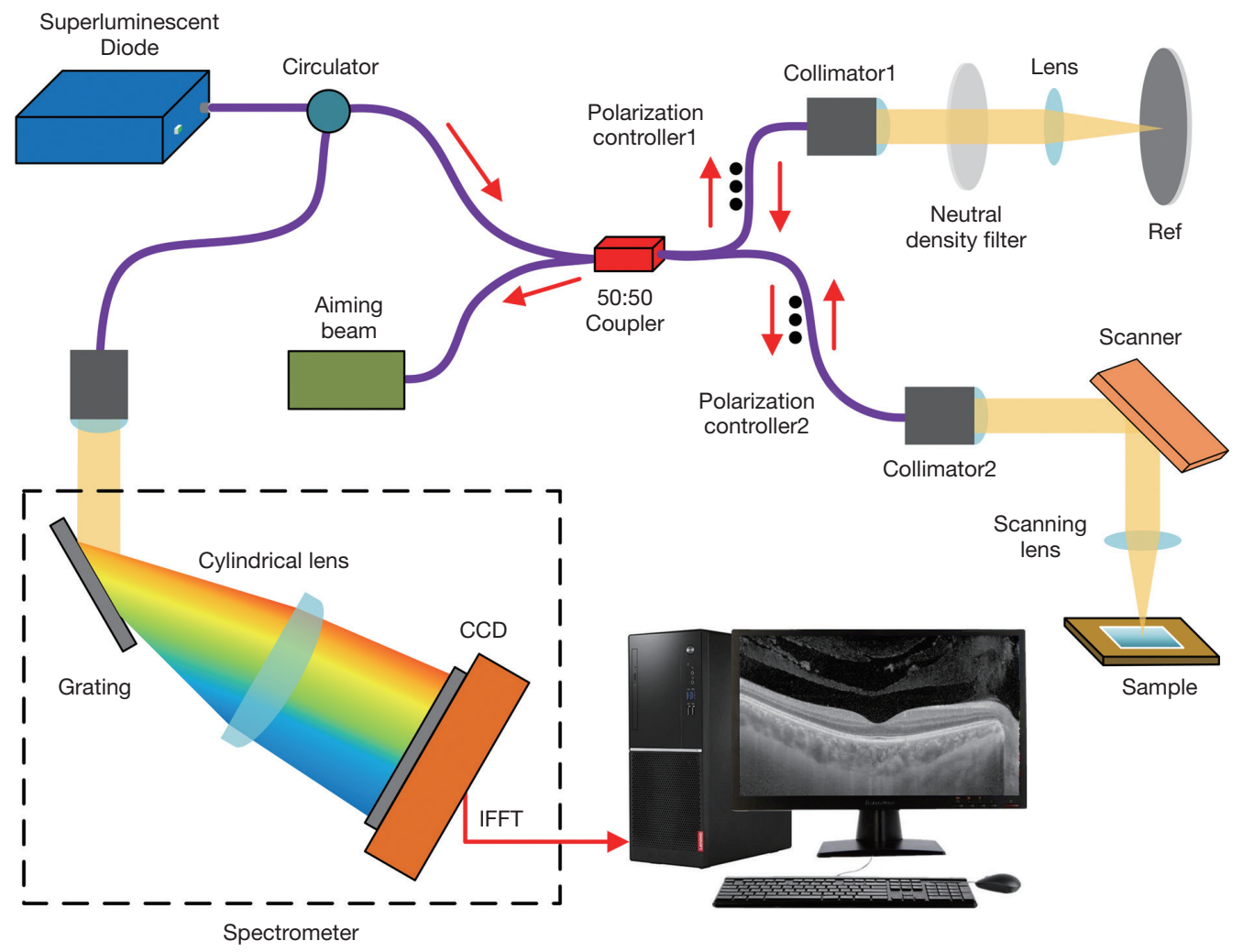

Figure 1 Original schematic diagram of optical coherence tomography.

images of intestinal tissues were examined by experienced gastroenterologists and OCT technicians to identify suspicious lesions. These technicians were trained on other OCT samples with known histopathological dysplasia or cancer diagnosis and blinded to the histological evaluation and other information.

\section{Histological analysis}

Once suspicious lesions were identified by OCT, pathological sections were taken from the same segment and evaluated to identify colorectal dysplasia (which was further classified as mild, moderate, or severe) or cancer in the sample. Other intestinal segments were taken from regions where no lesions were seen on the OCT images and evaluated by pathological examination and classification. The tissue samples were fixed in $4 \%$ paraformaldehyde for $24 \mathrm{~h}$. Then, the tissues were dehydrated, made transparent, dipped in wax, embedded in paraffin blocks, cut into $4 \mu \mathrm{m}$ sections, and stained with hematoxylin and eosin (HE). Histological images of each sample were captured under a microscope and evaluated by two experienced pathologists in a blinded manner. All images were numbered, and the OCT images and HE-stained pathological sections were matched according to their morphological and structural characteristics.

\section{Histological classification criteria for colorectal lesions}

HE-stained colorectal lesions were classified histologically according to the WHO/Vienna (11) classification criteria for gastrointestinal epithelial neoplasms (Table 1). The pathological evaluation results were used as the goldstandard reference.

\section{Data analysis}

The OCT images were processed using Matlab software. Biomedical image analysis software (Image-Pro Plus 6.0.0260), followed by Image J software, were used to qualitatively analyze the submucosal morphological changes in the HE-stained sections and OCT images. The experimental data were processed using SPSS 21.0 software. Fisher's exact test was used to compare the positive rates of OCT and pathological evaluation $(\mathrm{P}<0.05$ was considered 
Table $1 \mathrm{WHO} /$ Vienna classification criteria and the characteristics of 2D- and 3D-OCT imaging for colorectal lesions

\begin{tabular}{|c|c|c|c|c|}
\hline Variable & Mild dysplasia & Moderate dysplasia & Severe dysplasia & Invasive cancer \\
\hline HE images & $<1 / 3$ & $1 / 3-2 / 3$ & $\begin{array}{l}>2 / 3 \text {, but not reaching } \\
\text { basement membrane }\end{array}$ & $\begin{array}{l}\text { Breaking through } \\
\text { basement membrane }\end{array}$ \\
\hline $\begin{array}{l}\text { 2D- and 3D-OCT } \\
\text { images }\end{array}$ & \multicolumn{4}{|c|}{$\begin{array}{l}\text { (I) With increasing grades of dysplasia to canceration: decreasing pixel intensities in the greyscale images, thickening } \\
\text { of the low-reflective layer, thinning of the super-reflective layer, and progressive disappearance of the stratified } \\
\text { structure. (II) Vertical stripes occurred only in dysplasia tissues }\end{array}$} \\
\hline
\end{tabular}

$\mathrm{HE}$, hematoxylin and eosin; OCT, optical coherence tomography.

Table 2 Animal and tumor characteristics (unit: piece)

\begin{tabular}{|c|c|c|c|c|c|c|}
\hline Variable & Control & Mild dysplasia & Moderate dysplasia & Severe dysplasia & Invasive cancer & Total \\
\hline Survival of mice & 12 & 4 & 3 & 6 & 3 & 28 \\
\hline HE images & 12 & 4 & 3 & 6 & 3 & 28 \\
\hline OCT images & 3996 & 1332 & 999 & 1998 & 999 & 9324 \\
\hline
\end{tabular}

$\mathrm{HE}$, hematoxylin and eosin; OCT, optical coherence tomography.

statistically significant). The Youden index, diagnostic efficiency, sensitivity, specificity, positive predictive value, and negative predictive value of OCT imaging were calculated with respect to the pathological evaluation results as the reference standard.

\section{Results}

\section{Animal and tumor characteristics}

Table 2 shows the survival and neoplasia formation observed in the mice, the histological classification results of the colorectal lesions, and the numbers of OCT and HEstaining images captured. Several of the AOM/DSS model mice died. However, based on the preliminary experiments of this study, the survival rate was acceptable and adequate. A representative OCT image was selected from the 333 OCT images captured from each intestinal segment for subsequent analysis and compared with the single HEstaining image captured from each mouse.

\section{Analysis of corresponding HE-stained tissue sections and OCT images}

Tissue with mild dysplasia cannot be distinguished from normal colon tissue by naked eye, but moderate to severe dysplasia and invasive cancer with prominent lumps can be readily distinguished from normal colon tissue (Table 3). Tissues with increasing grades of dysplasia to cancer appear more heterogeneous, the mucosa becomes thicker, the architecture becomes more unorganized, and the glands become more irregular upon pathological examination. These characteristic pathological changes were manifested as changes in the low- and super-reflective layers in the OCT images. The 2D- and 3D-OCT images of tissues with increasing grades of dysplasia to cancer exhibited decreasing pixel intensities in the greyscale images, thickening of the low-reflective layer, thinning of the super-reflective layer, and progressive disappearance of the stratified structure (Tables 1 and 3). Dysplasia and cancer could be distinguished from normal colon tissues from either the HE-stained sections and OCT images. However, compared to pathological evaluation, the $2 \mathrm{D}$ - and $3 \mathrm{D}$-OCT images could not provide sufficient information to differentiate different degrees of dysplasia or distinguish dysplasia from cancerous tissues.

\section{Statistical analysis results}

Considering the pathological evaluation results as the reference standard, the diagnostic performance of OCT for distinguishing colorectal cancer and dysplasia from normal colon tissue was characterized by an overall sensitivity of 
Table 3 Tissue samples and corresponding HE-stained tissue sections and OCT images

\begin{tabular}{|c|c|c|c|c|c|}
\hline Variable & Normal & Mild dysplasia & Moderate dysplasia & Severe dysplasia & Invasive cancer \\
\hline \multicolumn{6}{|c|}{ HE staining } \\
\hline \multicolumn{6}{|l|}{ 2D-OCT } \\
\hline \multicolumn{6}{|l|}{ 3D-OCT } \\
\hline
\end{tabular}

$\mathrm{HE}$, hematoxylin and eosin; OCT, optical coherence tomography; 2D-OCT, two-dimensional optical coherence tomography; 3D-OCT, three-dimensional optical coherence tomography.

$87.50 \%$, specificity of $75.00 \%$, negative predictive value of $81.82 \%$, positive predictive value of $82.35 \%$, diagnostic efficiency of $82.14 \%$, and Youden index of $62.50 \%$. There was no significant difference between the diagnostic performance with OCT and pathological section evaluation $(\mathrm{P}=1.000,>0.05)$ (Tables 4 and 5).

\section{Discussion}

Currently, the standard clinical diagnosis of colorectal lesions relies almost exclusively on colonoscopy with biopsy. However, it cannot provide a sufficient positive detection rate or diagnostic accuracy for colorectal cancer and dysplasia. Therefore, there is an urgent need to develop a non-invasive imaging technique that would provide more sensitive imaging capabilities and enable a qualitative analysis for areas that are suspected to be cancerous to guide targeted biopsies. Fortunately, given the advantages of submucosal imaging, OCT imaging has been successfully applied to image a broad spectrum of malignancies, including those arising in the breast, brain, bladder, skin, and oral cavity as well as the gastrointestinal, respiratory system, and reproductive systems (12). OCT has broad potential applications, especially in the field of gastrointestinal oncology, including cancer screening, biopsy guidance, intraoperative imaging, posttreatment surveillance $(12,13)$. The application of OCT in gastrointestinal disease $(8,14)$ makes the above objective achievable. Surprisingly, our study showed that, compared with conventional white light endoscopy, OCT provides a superior positive detection rate and diagnostic accuracy. Colorectal dysplasia or cancer could be distinguished from normal tissues based on the OCT images. However, the simple morphological characteristics in the OCT images 
Table 4 Comparison of OCT and pathological results in the diagnosis of colorectal cancer and dysplasia

\begin{tabular}{lccc}
\hline \multirow{2}{*}{ OCT results } & \multicolumn{1}{c}{ HE-stained pathological evaluation results } & Normal & Total \\
\cline { 2 - 3 } & Colorectal cancer and dysplasia & 3 & 17 \\
Colorectal cancer and dysplasia & 14 & 9 & 11 \\
Normal & 2 & 12 & 28 \\
\hline
\end{tabular}

$\mathrm{HE}$, hematoxylin and eosin; OCT, optical coherence tomography.

Table 5 Statistical indicators from the experimental evaluation of diagnostic capabilities with OCT

\begin{tabular}{ll}
\hline Variable & Result \\
\hline Sensitivity & $87.50 \%$ \\
Specificity & $75.00 \%$ \\
Positive predicting value & $82.35 \%$ \\
Negative predicting value & $81.82 \%$ \\
Youden index & $62.50 \%$ \\
Diagnostic efficiency & $82.14 \%$ \\
\hline
\end{tabular}

OCT, optical coherence tomography.

were not sufficient to distinguish dysplasia from cancer.

Comparing the OCT observations with the pathological evaluation results (used as the gold standard) for tissues having different degrees of dysplasia and cancer tissues, our results demonstrated that OCT was highly sensitive and specific for the early detection of colorectal dysplasia and cancer. This finding suggests the clinical feasibility of OCT for imaging gastrointestinal lesions. Compared with conventional white light endoscopy, in which the diagnostic sensitivity is generally around $47-53 \%$ (15), OCT was superior in terms of the positive detection rate and the diagnostic accuracy. Compared with narrowband imaging, which is the current gold standard for endoscopic visualization of vessel networks, OCT can achieve a higher resolution that approaches the cellular scale $(4-20 \mu \mathrm{m})$ with a deeper penetration depth that is comparable with that of conventional biopsy (2-3 mm) $(16,17)$. In particular, OCT can be combined with optical coherence tomography angiography (OCTA) to visualize subsurface microvasculature, provide volumetric $3 \mathrm{D}$ datasets, and simultaneously display detailed morphological and functional information (18). Therefore, OCT has the potential to be a powerful technique for the early detection of colorectal dysplasia or cancer.
As with other new imaging modalities, early investigations using OCT were focused on the structural features corresponding to pathology. Previous studies have confirmed that the morphological stratification of the intestinal wall is consistent between OCT imaging and HE staining $(4,19)$. The early diagnosis of colorectal dysplasia and cancer in HE-stained tissue sections is mainly based on the degrees of proliferation and heteromorphism of the mucosal cells, mucosal incrassation, and structural characteristics. In this study, the OCT images showed that the mucosa was super-reflective, and the submucosa and muscularis propria were low-reflective. The early colorectal cancer and dysplasia in the OCT images were characterized by different degrees of abnormality in the mucosal texture, the absence of stratified mucosal structures, and differences in the grayscale intensities of the super- and low-reflective layers compared with normal tissue, which is consistent with the findings of previous studies (8-10).

As this study suggested, as the grade of colorectal dysplasia and cancer increases, the major pathological correlates of mucosal thickening and grayscale pixel intensity changes in OCT images were increases in the numbers of mucosal epithelial and inflammatory cells, unbalancing of the cellular nucleus-to-cytoplasm ratio, increasing irregularity in the arrangement of glands, and proliferation and deformation of mucosal vessels. All of these factors contributed to the irregularity in the scattering direction of the near-infrared light captured by OCT, leading to a reduction in the greyscale pixel intensities of the mucosal super-reflective layer. Another factor contributing to this effect, though likely to a lesser degree, was that the mucosal interstitial tissues grew along the vertical axis of the mucosa.

Our findings showed that dysplasia and cancer could be distinguished from normal colon tissue on OCT images. As the pathological grade of the dysplasia increases until canceration, the changes in thickness and the grayscale pixel intensities of the super- and low-reflective layers in 2D- and 3D-OCT images became increasingly prominent. Notably, 
once dysplasia formed, the grayscale pixel intensities of the super-reflective layer in $2 \mathrm{D}$ - and $3 \mathrm{D}$-OCT images were lower than those of a normal colon. As the lesions progressed, the increase in the mucosal thickness and loss of stratified structures made the difference more pronounced, facilitating the differentiation between dysplasia and normal tissue. Further, the presence of darker vertical stripes in the low-reflective layer of the tissue suggested that the lesion was likely to be dysplasia, as this feature was observed only in dysplasia tissues.

In addition, the OCT images of cancer tissues exhibited obvious mucosal-structure abnormalities, increased mucosal thicknesses, and narrowed and decreased grayscale pixel intensities in the super-reflective layer compared with normal colon tissues. Masses with different degrees of protrusion had somewhat narrowed super-reflective layers on 3D-OCT images, as well as relatively low greyscale pixel intensities and apparent abnormalities in their mucosal structures compared with normal tissues; these features were key distinguishing characteristics. Therefore, the OCT images should be interpreted primarily based on the changes in the stratified mucosal structures and the super-reflective layer, as these morphological changes are associated with early colorectal cancer lesions.

$3 \mathrm{D}$ images were obtained by performing 2D scan patterns at different transverse planes (20). The advantages of $3 \mathrm{D}-\mathrm{OCT}$ were that it provided more information about the $3 \mathrm{D}$ structures of lesions than 2D-OCT, including more overall scattering information. Thus, when the imaging angle and information obtained of 2D-OCT are unsatisfactory, 3D-OCT could make up for the lack of scattering information. Further, when it is difficult to determine the nature of a lesion from 2D-OCT images, 3D-OCT images can be helpful for further qualitative diagnosis as the visualization of morphological abnormalities is more intuitive than with $2 \mathrm{D}-\mathrm{OCT}$.

Unfortunately, no apparent differences were observed between OCT images of tissues with different degrees of dysplasia or between dysplasia and cancer tissues, whether 2D- or 3D-OCT images. Firstly, comparing tissues with mild to severe dysplasia, the changes in the mucosal thickness and the grayscale pixel intensities in the 2D- and 3D-OCT images were not significant enough to make definitive distinctions, because of their similar scattering characteristics. Secondly, comparing cancer tissues with those having different degrees of dysplasia, both 2Dand 3D-OCT images revealed more disordered mucosa textures, loss of stratified structures, and narrowed super- reflective layers. However, simple morphological changes in the OCT images were not sufficient to distinguish dysplasia from cancerous tissues. Therefore, with the currently available OCT devices, it would be premature to claim that OCT could play a role in the real-time differential diagnosis of abnormal tissues in vivo. Thus, excisional biopsy and histology are still irreplaceable.

Fortunately, although it was challenging to distinguish dysplasia from cancer, non-lesion areas could be readily excluded. Moreover, OCT can enable real-time qualitative analysis and guide targeted biopsies of lesions suspected to be early colorectal cancer. This application is expected to reduce sampling errors and the number of biopsies needed, thus increasing the diagnostic yield of endoscopy and histology, which is especially valuable in situations where conventional biopsy is ineffective or where biopsies may lead to complications. Therefore, OCT might be applied in screening high-risk patient populations in the future.

While OCT possesses excellent diagnostic potential and is relatively simple to implement, the wavelength of $1,000 \mathrm{~nm}$ likely limits the depth of imaging (although no problems were encountered in mice) and currently there are other technical capacities. Moreover, OCT is mainly used for qualitative research at present, rather than quantitative analysis. Defined OCT parameters histologically proven to detect colonic dysplasia have not been identified. However, our research results prompt further related research and exploration into OCT-based indicators that may potentially assist the diagnosis of colorectal cancer and dysplasia. In the future, OCT can be combined with other techniques [such as inverse spectroscopic OCT (21), micro-optical OCT (22), Raman OCT (23), and fluorescence-guided OCT (24)], and the complementary optical information could be leveraged to realize "optical biopsies" comparable to pathological diagnosis. Hence, the advantages of OCT are irreplaceable, and its prospects are vast.

\section{Conclusions}

OCT is a promising non-invasive imaging technique that may improve the positive detection rate and diagnostic accuracy of early colorectal dysplasia and cancer, and may ultimately enable real-time qualitative analysis and guide targeted biopsies.

\section{Acknowledgments}

We thank Stephanie Knowlton, PhD, from Liwen Bianji, 
Edanz Editing China (www.liwenbianji.cn/ac), for editing the English text of a draft of this manuscript.

Funding: The study was supported by the Scientific Education Research Fund for middle-aged and young teachers of the ministry of Fujian Province (JK2017021), the Training Fund for middle-aged and young talent in Fujian Province (2017-ZQN-41), the Joint Funds for the innovation of science and technology in Fujian Province (2017Y9048), and the Fujian Natural Science Foundation (2018J01314).

\section{Footnote}

Conflicts of Interest: All authors have completed the ICMJE uniform disclosure form (available at http://dx.doi. org/10.21037/qims-20-13). The authors have no conflicts of interest to declare.

Ethical Statement: Experiments were performed under a project license (No.: FJMU IACUC 2019-0069) granted by the Institutional Animal Care and Use Committee (IACUC) of Fujian Medical University, in compliance with FJMU IACUC institutional guidelines for the care and use of animals.

Open Access Statement: This is an Open Access article distributed in accordance with the Creative Commons Attribution-NonCommercial-NoDerivs 4.0 International License (CC BY-NC-ND 4.0), which permits the noncommercial replication and distribution of the article with the strict proviso that no changes or edits are made and the original work is properly cited (including links to both the formal publication through the relevant DOI and the license). See: https://creativecommons.org/licenses/by-nc-nd/4.0/.

\section{References}

1. Deegan AJ, Mandell SP, Wang RK. Optical coherence tomography correlates multiple measures of tissue damage following acute burn injury. Quant Imaging Med Surg 2019;9:731-41.

2. Jäckle S, Gladkova N, Feldchtein F, Terentieva A, Brand B, Gelikonov G, Gelikonov V, Sergeev A, FritscherRavens A, Freund J, Seitz U, Soehendra S, Schrödern N. In Vivo Endoscopic Optical Coherence Tomography of the Human Gastrointestinal Tract-Toward Optical Biopsy. Endoscopy 2000;32:743-49.

3. Hoffman A, Manner H, Rey JW, Kiesslich R. A guide to multimodal endoscopy imaging for gastrointestinal malignancy—an early indicator. Nat Rev Gastroenterol Hepatol 2017;14:421-34.

4. Neufert C, Becker C, Neurath MF. An inducible mouse model of colon carcinogenesis for the analysis of sporadic and inflammation-driven tumor progression. Nat Protoc 2007;2:1998-2004.

5. Fan Y, Xia Y, Zhang X, Sun Y, Tang J, Zhang L, Liao H. Optical coherence tomography for precision brain imaging, neurosurgical guidance and minimally invasive theranostics. Biosci Trends 2018;12:12-23.

6. Tang Q, Liang CP, Wu K, Sandler A, Chen Y. Real-time epidural anesthesia guidance using optical coherence tomography needle probe. Quant Imaging Med Surg 2015;5:118-24.

7. Tang Q, Tsytsarev V, Liang CP, Akkentli F, Erzurumlu RS, Chen Y. In Vivo Voltage-Sensitive Dye Imaging of Subcortical Brain Function. Sci Rep 2015;5:17325.

8. Pitris C, Jesser C, Boppart SA, Stamper D, Brezinski ME, Fujimoto JG. Feasibility of optical coherence tomography for high-resolution imaging of human gastrointestinal tract malignancies. J Gastroenterol 2000;35:87-92.

9. van Manen L, Dijkstra J, Boccara C, Benoit E, Vahrmeijer AL, Gora MJ, Mieog JSD. The clinical usefulness of optical coherence tomography during cancer interventions. J Cancer Res Clin Oncol 2018;144:1967-90.

10. Pfau PR, Sivak MV Jr, Chak A, Kinnard M, Wong RC, Isenberg GA, Izatt JA, Rollins A, Westphal V. Criteria for the Diagnosis of Dysplasia by Endoscopic Optical Coherence Tomography. Gastrointest Endosc 2003;58:196-202.

11. Huang SF. WHO/Vienna classification of gastrointestinal epithelial neoplasms with emphasis on colorectal cancer prelesion. Chin J pathology 2005;34:540-1.

12. Wang J, Xu Y, Boppart SA. Review of optical coherence tomography in oncology. J Biomed Opt 2017;22:1-23.

13. Tsai TH, Leggett CL, Trindade AJ, Sethi A, Swager AF, Joshi V, Bergman JJ, Mashimo H, Nishioka NS, Namati E. Optical coherence tomography in gastroenterology: a review and future outlook. J Biomed Opt 2017;22:1-17.

14. Testoni PA, Mangiavillano B. Optical coherence tomography in detection of dysplasia and cancer of the gastrointestinal tract and bilio-pancreatic ductal system. World J Gastroenterol 2008;14:6444-52.

15. Iftimia N, Iyer AK, Hammer DX, Lue N, Mujat M, Pitman M, Ferguson RD, Amiji M. Fluorescence-guided optical coherence tomography imaging for colon cancer screening: a preliminary mouse study. Biomed Opt Express 
2012;3:178-91.

16. Kobayashi K, Izatt JA, Kulkarni MD, Willis J, Sivak MV Jr. High-resolution cross-sectional imaging of the gastrointestinal tract using optical coherence tomography: preliminary results. Gastrointest Endosc 1998;47:515-23.

17. Ding J, Lin JW, Li Q, Chen XP, Chen WQ, Zhang QK, He SS,Wu T, Wang CD, Zhong SC, Li D.

Optical coherent tomography to evaluate the degree of inflammation in a mouse model of colitis. Quant Imaging Med Surg 2020;10:945-57.

18. Wurster LM, Kretschmer S, Jäger J, Placzek F, Ginner L, Drexler W, Ataman Ç, Leitgeb RA, Zappe H. Comparison of optical coherence tomography angiography and narrowband imaging using a bimodal endoscope. J Biomed Opt 2019;25:1-5.

19. Hosokawa O, Watanabe K, Hatorri M, Douden K, Hayashi H, Kaizaki Y. Detection of gastric cancer by repeat endoscopy within a short time after negative examination. Endoscopy 2001;33:301-5.

20. Tsai TH, Fujimoto JG, Mashimo H. Endoscopic Optical Coherence Tomography for Clinical Gastroenterology.

Cite this article as: Ding J, Li Q, Lin J, He S, Chen W, He Q, Zhang Q, Chen J, Wu T, Zhong S, Li D. Optical coherence tomography for the early detection of colorectal dysplasia and cancer: validation in a murine model. Quant Imaging Med Surg 2021;11(1):371-379. doi: 10.21037/qims-20-13
Diagnostics 2014;4:57-93.

21. Spicer GL, Azarin SM, Yi J, Young ST, Ellis R, Bauer GM, Shea LD, Backman V. Detection of extracellular matrix modification in cancer models with inverse spectroscopic optical coherence tomography. Phys Med Biol 2016;61:6892-904.

22. Luo Y, Cui D, Yu X, Bo E, Wang X, Wang N, Braganza CS, Chen S, Liu X, Xiong Q, Chen S, Chen S, Liu L. Endomicroscopic optical coherence tomography for cellular resolution imaging of gastrointestinal tracts. J Biophotonics 2018;11:e201700141.

23. Ashok PC, Praveen BB, Bellini N, Riches A, Dholakia K, Herrington CS. Multi-modal approach using Raman spectroscopy and optical coherence tomography for the discrimination of colonic adenocarcinoma from normal colon. Biomed Opt Express 2013;4:2179-86.

24. Iftimia N, Iyer AK, Hammer DX, Lue N, Mujat M, Pitman M, Ferguson RD, Amiji M. Fluorescence-guided optical coherence tomography imaging for colon cancer screening: a preliminary mouse study. Biomed Opt Express 2012;3:178-91. 